\title{
Comparison between immediately and next day post RF ablation MRI in patients with atrial fibrillation
}

\author{
Eugene Kholmovski ${ }^{1,2^{*}}$, Sathya Vijayakumar ${ }^{1,2}$, Christopher McGann ${ }^{2}$, Nassir F Marrouche ${ }^{2}$ \\ From 15th Annual SCMR Scientific Sessions \\ Orlando, FL, USA. 2-5 February 2012
}

\section{Background}

Atrial fibrillation (AF) is the most common cardiac arrhythmia affecting more than 5 million people in North America and Europe. Radio-frequency ablation (RFA) is effective in symptomatic, drug refractory AF. Reported success rates of the procedure vary significantly with AF recurrences ranging from $25-40 \%$. The transmural extent of left atrial (LA) wall injury is difficult to assess with conventional electro-physiological (EP) measurements. Late gadolinium enhancement (LGE) and double inversion recovery T2-weighted TSE (DIR-T2w-TSE) and HASTE have been proposed to evaluate acute LA wall injury. In this study, we examine the evolution of acute $(<30$ hours after RFA) atrial lesions in LGE and DIR-T2w-TSE MRI with time after the procedure. The main goals of this project are to study changes in acute lesion appearance with time after RF ablation and to determine the time interval after ablation when MRI achieves the best visibility of acute LA lesions.

\section{Methods}

From January 2010 to September 2011, 224 AF patients who underwent pulmonary vein isolation and debulking of the septal and posterior walls under EAM guidance (CARTO, Biosense Webster) were imaged on a 3 Tesla MR scanner (Verio, Siemens Healthcare). Typical time interval between the conclusion of the ablation procedure and patient in the scanner was less than an hour. MRI studies were performed to rule out procedure complications and assess the extent of injury to LA wall. The study protocol included DIR-

'UCAIR, Department of Radiology, University of Utah, Salt Lake City, UT, USA Full list of author information is available at the end of the article
T2w-TSE, contrast enhanced MR angiography (Multihance, $0.1 \mathrm{mmol} / \mathrm{kg}$ ), and 3D LGE scans. Ten patients underwent an additional MRI study on the next day after ablation (22.0 \pm 2.6 hours) to follow-up on significant enhancement of the anterior wall of the esophagus in vicinity of LA wall, detected by immediately post-ablation LGE-MRI. Contrast-to-noise ratio (CNR) between $\mathrm{LA}$ lesion and blood $\left(\mathrm{CNR}_{\mathrm{LB}}\right)$ and between LA lesion and normal myocardium $\left(\mathrm{CNR}_{\mathrm{LM}}\right)$ were evaluated for these patients using immediately and next day post-ablation DIR-T2w-TSE and LGE images. Signal intensity for LA lesion/blood/normal myocardium was measured in LA posterior wall/LA cavity/LV wall, correspondingly. Standard deviation of noise was assumed to be equal to standard deviation of blood signal in the LA cavity.

\section{Results}

Quantitative analysis (Table 1) and visual inspection (Fig.1) of the DIR-T2w-TSE and LGE images indicate that contrast between ablated regions of LA wall and the other tissues is significantly higher in immediately post-ablation images than in the next day post-ablation images.

Table 1 CNR $_{L M}$ and CNR $_{L B}$ in immediately and next day post-ablation DIR-T2w-TSE and LGE-MRI

\begin{tabular}{ccccccc}
\hline & \multicolumn{3}{c}{ DIR-T2w-TSE } & \multicolumn{3}{c}{ LGE-MRI } \\
\cline { 2 - 7 } & $\begin{array}{c}\text { Immed. } \\
\text { Post }\end{array}$ & Next Day & $\begin{array}{c}\mathrm{p}^{-} \\
\text {value }\end{array}$ & $\begin{array}{c}\text { Immed. } \\
\text { Post }\end{array}$ & Next Day & $\begin{array}{c}\mathrm{p}^{-} \\
\text {value }\end{array}$ \\
\hline CNR $_{\mathrm{LM}}$ & $17.53 \pm 3.04$ & $8.90 \pm 4.35$ & 0.001 & $16.47 \pm 2.17$ & 11.92 & 0.0005 \\
& & & & & \pm 2.62 & \\
CNR $_{\text {LB }}$ & $23.24 \pm 4.96$ & 13.76 & 0.005 & $9.17 \pm 2.36$ & $3.75 \pm 2.52$ & 0.0001 \\
& & \pm 5.38 & & & & \\
\hline
\end{tabular}



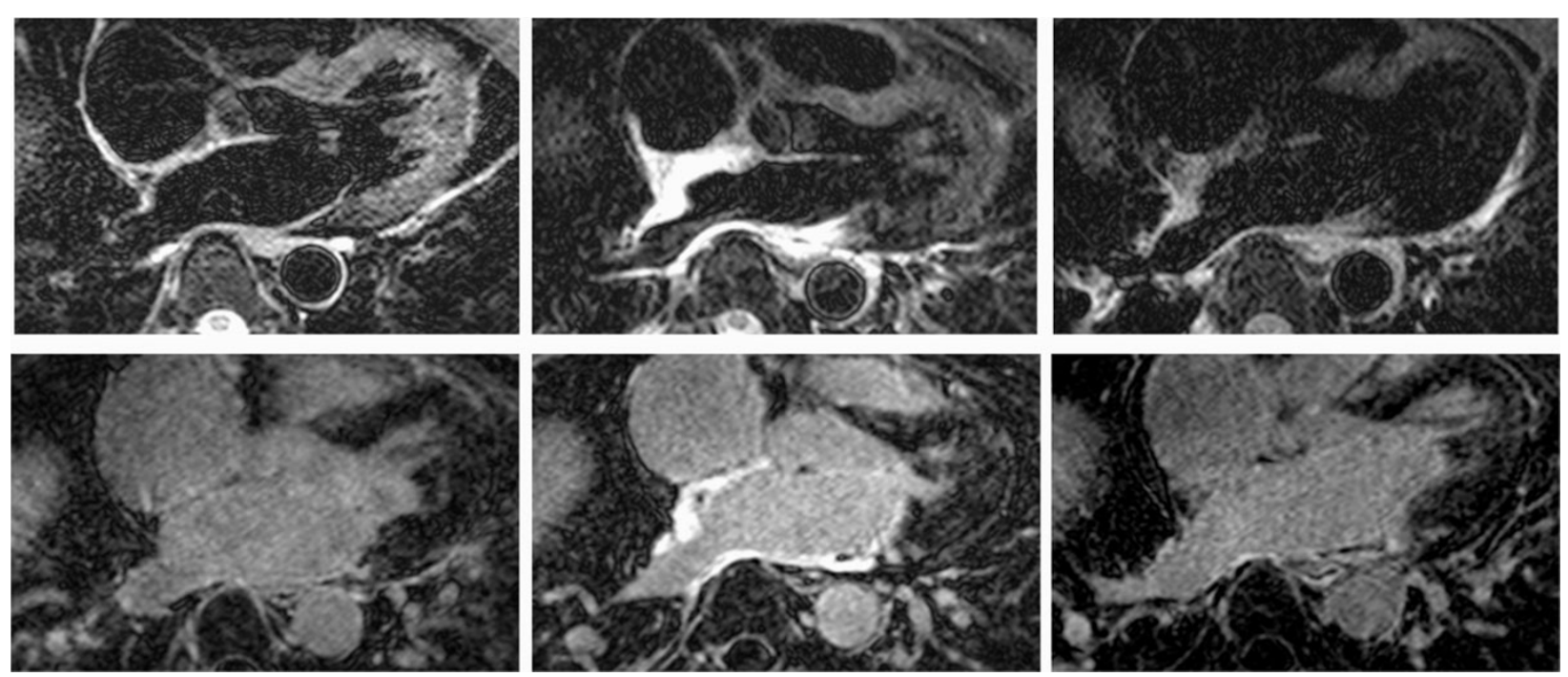

Figure 1 DIR-T2W-TSE (top row) and LGE (low row) images of AF patient. Left column - pre-ablation, middle column - immediately postablation, right column - next day post-ablation. Notice high contrast between ablated regions of LA wall (posterior wall, septum, ostia of right posterior PV) and the other tissues in immediately post-ablation DIR-T2W-TSE and LGE images.

\section{Conclusions}

The presented results indicate a fast physiological response of LA wall to injury. Edema is noticeably reduced 24 hours after RF ablation. Kinetics of contrast agent in injured regions changes considerably during the first 24 hours after RF ablation. Our results demonstrate that MRI study should be performed as soon as possible after conclusion of the RFA procedure to achieve high contrast between ablated LA wall and the other tissues.

\section{Funding}

Ben B. and Iris M. Margolis Foundation.

\section{Author details}

'UCAIR, Department of Radiology, University of Utah, Salt Lake City, UT, USA.

${ }^{2}$ CARMA Center, University of Utah, Salt Lake City, UT, USA.

Published: 1 February 2012

doi:10.1186/1532-429X-14-S1-P200

Cite this article as: Kholmovski et al:: Comparison between immediately and next day post RF ablation MRI in patients with atrial fibrillation. Journal of Cardiovascular Magnetic Resonance 2012 14(Suppl 1):P200.

\section{Submit your next manuscript to BioMed Central} and take full advantage of:

- Convenient online submission

- Thorough peer review

- No space constraints or color figure charges

- Immediate publication on acceptance

- Inclusion in PubMed, CAS, Scopus and Google Scholar

- Research which is freely available for redistribution 